\title{
Anakinra in Still's disease: a profile of its use
}

\author{
Katherine A. Lyseng-Williamson ${ }^{1}$
}

Published online: 31 October 2018

(C) Springer Nature 2018, corrected publication 2018

\begin{abstract}
The EU indication for anakinra has been extended to include Still's disease, a serious rare inflammatory disorder of unknown aetiology that comprises adult-onset Still's disease (AOSD) and systemic juvenile idiopathic arthritis (SJIA). As activated interleukin-1 pathways are associated with the systemic manifestations of these disorders, targeted treatment with anakinra, an interleukin-1 inhibitor, has been investigated. Across clinical and real-world studies in patients with AOSD and SJIA, treatment with anakinra achieved clinical remission/response, provided rapid and sustained improvements in systemic and laboratory manifestations, and allowed the use of corticosteroid- and disease-modifying anti-rheumatic drugs (DMARD) to be reduced or discontinued. The safety profile of anakinra in the treatment of Still's disease is consistent with that in its other approved indications.
\end{abstract}

\section{Adis evaluation of anakinra in Still's disease}

Neutralizes the inflammatory effects of interleukin-1

Rapidly leads to clinical responses, with sustained improvements in systemic and laboratory manifestations in patients with AOSD and SJIA

Allows the use of corticosteroids and DMARDs to be reduced or discontinued, thereby reducing the risk of adverse drug reactions associated with such treatment

Well tolerated, with injection-site reactions being the most common treatment-related adverse events

Precautions should be taken to reduce the likelihood of injection-site reactions and other events

\section{What is the rationale for using anakinra in Still's disease?}

Still's disease is a serious inflammatory disorder that presents as adult-onset Still's disease (AOSD) [1-5] or, in children aged $\leq 16$ years, as systemic juvenile idiopathic

Katherine A. Lyseng-Williamson

dtp@adis.com

1 Springer, Private Bag 65901, Mairangi Bay, 0754 Auckland, New Zealand arthritis (SJIA) [6, 7]. The aetiology and pathogenesis of these conditions are largely unknown, with both presenting with heterogeneous non-specific symptoms, such as fever, skin rash and haematological disturbances, and both being associated with long-term disability, increased morbidity and mortality, and reduced health-related quality of life [1, $3-6,8]$. Results of gene-expression analyses suggest that AOSD and SJIA are expressions of a continuum of a single disease that differ by their time of onset, and that susceptibility to these disorders is associated with variations in certain genes [9].

Based on their signs and symptoms, patients with Still's disease may have primarily systemic disease [associated with high fever, high levels of C-reactive protein (CRP), liver enzymes, ferritin, and interleukin (IL)- 1 and IL-18, and elevated erythrocyte sedimentation rates (ESR)] or chronic articular disease [associated with arthritis, a lack of fever in some patients, and high levels of tumour necrosis factor (TNF)- $\alpha$, and IL-6 and IL-17] [1, 2, 4-6]. Although arthritis may not be present for months or even years in patients with SJIA, the extent of joint involvement, as well as the persistence of the systemic symptoms, correlate with patients' overall prognosis [6].

Potentially fatal complications of AOSD and SJIA include pulmonary complications (e.g. pulmonary arterial hypertension, acute respiratory failure, interstitial lung disease and alveolar proteinosis), cardiac complications (e.g. myocarditis), disseminated intravascular coagulopathy, thrombotic thrombocytopenic purpura, and 
macrophage activation syndrome (MAS; a type of secondary haemophagocytic lymphohistiocytosis) [4, 8, 10-13]. MAS is a serious life-threatening complication of Still's disease and other rheumatological diseases $[12,13]$. It results from uncontrolled overproduction of inflammatory cytokines, including IL-1, and is related to the mutation of specific genes $[12,13]$.

Empirical treatment of AOSD and SJIA has involved the use of NSAIDs, corticosteroids and disease-modifying anti-rheumatic drugs (DMARDs), such as methotrexate, ciclosporin, azathioprine, sulfasalazine, leflunomide and intravenous immunoglobulin (IVIg) [1, 3-6, 14]. With the discovery of the mediators of the inflammatory cascade underlying Still's disease and the development of biological DMARDs (bDMARDs), it is now possible to target treatment against the key inflammatory cytokines involved. For example, patients with systemic AOSD or SJIA have a preferential response to IL-1 inhibitors, whereas those with chronic articular AOSD have a preferential response to TNF- $\alpha$ inhibitors, as these conditions are associated with high levels of the respective cytokines $[1,2,6]$.

Anakinra $\left(\right.$ Kineret $\left.^{\circledR}\right)$ is a human IL-1 receptor antagonist produced in Escherichia coli cells by recombinant DNA technology [15]. Anakinra competitively inhibits IL- $1 \alpha$ and IL- $1 \beta$ from binding to the IL- 1 type I receptor, thereby neutralizing the activity of these key mediators of immune and inflammatory processes $[1,2,6,16]$. This review discusses the use of anakinra as recently approved to treat Still's disease, including SJIA and AOSD, in the EU [15]. It focuses on the results of key clinical trials, as well as relatively recent fully published observational studies in $\geq 20$ patients, meta-analyses and literature reviews. A discussion of the use of anakinra as previously approved to treat rheumatoid arthritis (RA) and cryopyrin-associated periodic syndromes (CAPS) is beyond the scope of this article.

\section{How should anakinra be used in the treatment of Still's disease?}

Anakinra is approved to treat Still's disease in adult and paediatric patients aged $\geq 8$ months with a body weight of $\geq 10 \mathrm{~kg}$, including those with AOSD or SJIA [15]. Patients should have either active systemic features of moderate to high disease activity or continued disease activity after treatment with NSAIDs or corticosteroids. Anakinra can be given as monotherapy or in combination with other anti-inflammatory drugs and DMARDs. Table 1 provides a summary of the administration, use in special populations and potentially clinically relevant drug interactions of anakinra as approved in the EU [15]. Consult local information for further details.

\section{What is the efficacy of anakinra in adult-onset Still's disease?}

\section{Versus DMARDs in an open-label trial}

Treatment with subcutaneous anakinra $100 \mathrm{mg} / \mathrm{day}$ had beneficial effects in patients with refractory AOSD, but its efficacy did not generally differ to that with DMARD treatment in an open-label, randomized, controlled trial (RCT) and its open-label extension [18]. This key study included 22 patients with AOSD who had received treatment with a corticosteroid and possibly a DMARD for $\geq 2$ months prior to randomization and who were considered to be refractory to such treatment [i.e. active AOSD despite treatment with corticosteroids (equivalent to $\geq 10 \mathrm{mg}$ / day of prednisolone) \pm concomitant DMARDs]. Of note, 60 patients (30 in each treatment group) were needed for statistical power, which differs considerably from the 22 patients enrolled in the study. Patients were randomized to receive 24 weeks of treatment with anakinra $(n=12)$ or a DMARD ( $n=10 ; 6$ received methotrexate, 2 received azathioprine and 2 received leflunomide); all patients initially received concomitant corticosteroid treatment equivalent to $\geq 10 \mathrm{mg} /$ day of prednisolone. Patients without improvement after week 24 in the randomized phase could switch to or add-on treatment with anakinra or a DMARD in a 28-week open-label extension for a total of 52 weeks' treatment $(n=17$; 9 patients who initially received anakinra and 8 who initially received a DMARD) [18].

Treatment with subcutaneous anakinra $100 \mathrm{mg} /$ day had beneficial effects in patients with refractory AOSD, but its efficacy did not generally differ to that with DMARD treatment (Table 2) [18]. The proportion of patients who achieved remission at weeks 4,8 and 24 did not differ to a significant extent between the anakinra and DMARD groups (Table 2) [18]. The only secondary endpoint with a significant treatment difference was the improvement from baseline in Medical Outcomes Study Short-Form 36 physical health summary scores (Table 2) [18]. The longer-term benefits of anakinra treatment were shown in the extension study, with half the patients receiving anakinra (alone or in combination) being in remission at week 52 (Table 2) [18].

\section{Retrospective observational studies}

Patients with refractory AOSD also responded to treatment with anakinra in relatively large $(n=41$ [19] and 141 [20]) observational studies (Table 2) [19, 20]. Anakinra recipients had rapid and sustained improvements in AOSD-associated clinical and laboratory manifestations $[19,20]$, with systemic manifestations generally being less 
Table 1 Administration of subcutaneous anakinra in the treatment of Still's disease in the EU [15]

\section{What is the approved indication for anakinra?}

In adults, adolescents, children and infants aged $\geq 8$ months with a body weight of $\geq 10 \mathrm{~kg}$ for the treatment of Still's disease, including systemic juvenile idiopathic arthritis and adult-onset Still's disease, with active systemic features of moderate to high disease activity, or in pts continued disease activity after treatment with NSAIDs or corticosteroids

Can be given as monotherapy or in combination with other anti-inflammatory and disease-modifying anti-rheumatic drugs

How is anakinra available and how should it be stored?

Availability

Ready-to-use, graduated, pre-filled single-use syringes containing $100 \mathrm{mg}$ of anakinra in $0.67 \mathrm{~mL}$ of solution for injection (allows doses of $20-100 \mathrm{mg}$ to be administered)

Storage

Refrigerate at $2-8{ }^{\circ} \mathrm{C}$ in the original packaging (to protect from light)

May be removed from the refrigerator for $12 \mathrm{~h}$ at temperatures $\leq 25^{\circ} \mathrm{C}$ (dispose of the syringe if it is not used by the end of this period)

How should anakinra be administered in pts with Still's disease?

Pts weighing $\geq 50 \mathrm{~kg}$

Dosage: $100 \mathrm{mg}$ once daily

Pts weighing $<50 \mathrm{~kg}$

Starting dosage of 1-2 mg/ $\mathrm{kg}$ once daily (may be increased to $4 \mathrm{mg} / \mathrm{kg} /$ day in children with an inadequate response)

Preparation before the injection Do not shake the syringe; allow the contents to come to room temperature (leave the syringe at room temperature for $\approx 30 \mathrm{~min}$ or hold it gently for a few minutes)

Method of administration

Subcutaneous injection in the abdomen (except for the area around the navel), top of thighs, upper outer areas of the buttocks and outer area of the upper arms

\section{How should anakinra be used in special populations?}

Pts aged $\geq 65$ years

No dose adjustment expected to be required

Use with caution (lack of data and increased risk of infection in this population)

Pts with renal impairment

Mild: no dose adjustment expected to be required

Moderate: use with caution

Severe or end-stage renal disease: consider administration every other day

Pts with hepatic impairment

Mild to moderate: no dose adjustment expected to be required

Severe: use with caution

Women who are pregnant or of

Avoid use (precautionary measure as data from the use of anakinra in pregnant women are limited)

child-bearing potential not using

contraception

Breast-feeding women

Discontinue breast-feeding during treatment (not known if anakinra is excreted in human milk)

What clinically relevant drug interactions may potentially occur with anakinra?

TNF- $\alpha$ antagonists

Concomitant use with anakinra is not recommended (may increase risk of serious infections or neutropenia without increased clinical benefit)

CYP substrates with a narrow therapeutic index (e.g. warfarin and phenytoin)

Consider therapeutic monitoring of the effect or concentration of such substrates and adjust substrate dosage if warranted (increased CYP levels can result from increased levels of cytokines, such as IL-1, during chronic inflammation; anakinra could normalize CYP levels as it is an IL-1 receptor antagonist)

Live vaccines

Do not administer concurrently with anakinra (more data required [17])

CYP cytochrome P450, IL interleukin, pt patient, TNF tumour necrosis factor

refractory to anakinra treatment than joint manifestations [19]. Moreover, treatment with anakinra may reduce the use of corticosteroids [19, 20] and/or DMARDs [20].

Treatment with anakinra after corticosteroid failure was also effective in patients with life-threatening AOSD, according to a case series [10]. In 20 patients with lifethreatening AOSD (first flare in $90 \%$ of patients), initial treatment with high-dose corticosteroids was effective (defined as being able to leave the intensive care unit without further treatment) in 10 patients (50\%) [10]. Secondline treatment with anakinra after corticosteroid failure was received by 5 patients; anakinra was effective in 4 of these patients (80\%), with a median time to response of 4.5 days (range 3-15) [10]. According to pooled data from the case series and its accompanying literature review in a total of 99 patients with AOSD with organ complications, anakinra was effective in $8 / 9$ patients (89\%), corticosteroids alone in $61 / 95$ (64\%), IVIg in $11 / 27(41 \%)$, ciclosporin in $13 / 18$ (72\%) and etoposide in 2/3 (67\%) [10].

\section{Meta-analyses and literature reviews}

A recent meta-analysis [21] and a literature review [22] have also shown anakinra to be an effective treatment of AOSD. 
Table 2 Efficacy of subcutaneous anakinra $2 \mathrm{mg} / \mathrm{kg}$ /day (maximum of $100 \mathrm{mg} /$ day) in treating patients with treatment-refractory adult-onse Still's disease in a randomized, open-label trial, its open-label extension [18] and recent retrospective studies [19, 20]

Open-label RCT of anakinra vs DMARDs in 22 pts [median AOSD duration before anakinra 1.2 vs 1.6 years (range 0.2-20)] [18]

More anakinra than DMARD recipients achieved remission ${ }^{\text {a }}$ at week 4 (50 vs 30\%), week 8 (58 vs 50\%; primary endpoint) and week 24 (50 vs $20 \%$ ), but the TDs were not significant at any of these time points

Changes from BL in SF-36 physical health summary scores favoured anakinra over DMARDs $(p=0.011)$

No significant TDs in changes from BL in SF-36 mental health summary scores, number of pts discontinuing corticosteroid treatment (3 vs 0), and CRP levels (reductions in both groups, with normal levels being achieved by week 8 , and maintained at week 24)

Mean prednisolone-equivalent dosages significantly $(p<0.05)$ decreased from BL in both the anakinra and DMARD groups $($ mean -10.8 vs $-10.5 \mathrm{mg}$ ); no significant TD

\section{Open-label extension of the RCT in 17 pts [18]}

Remission $^{\mathrm{a}}$ at week 52 was achieved by 7 of the 14 pts receiving anakinra alone or in combination, and 2 of the 3 pts receiving DMARDs

At week 52, 9 of the initial anakinra recipients were still receiving anakinra (8 as monotherapy and 1 in combination with methotrexate); of the 8 initial DMARD recipients, 3 continued to receive DMARDs, 2 switched to anakinra + methotrexate, and 1 each had switched to anakinra monotherapy, anakinra + leflunomide or infliximab monotherapy

\section{Retrospective study in 41 pts [median AOSD duration before anakinra 3.5 years (range 2-6)] [19]}

At 1 year, anakinra reduced the proportions of pts with systemic manifestations, including cutaneous rash (7.3\% from BL of 58.5\%), fever ( $14.1 \%$ from BL of $78.0 \%$ ), anaemia (9.8\% from BL of $56.1 \%$ ), lymphadenopathy (4.9\% from BL of $26.8 \%$ ), leucocytosis (14.6\% from BL of $65.9 \%$ ), splenomegaly/hepatomegaly (5.6\% from BL of $13.7 \%$ ) and pleuritis/pericarditis (2.4\% from BL of $19.5 \%$ )

Proportion of anakinra recipients with joint manifestations decreased to $41.5 \%$ at 1 year from BL of $87.8 \%$

Median prednisolone-equivalent dosage in anakinra recipients decreased to $5 \mathrm{mg} / \mathrm{day}$ (range 0-10) at 1 year from BL of $20 \mathrm{mg} / \mathrm{day}$

Anakinra provided rapid and sustained improvements from BL in laboratory parameters, including ESR, and CRP, haemoglobin, ferritin and leukocyte levels

Retrospective study in 140 pts (mean AOSD duration before anakinra 4.2 years) [20]

Proportion of pts still receiving anakinra decreased to $69.2 \%$ at 1 year and $49.3 \%$ during follow-up (mean treatment duration 3 years); treatment was switched to canakinumab in 4 pts

Of the 71 pts (50.7\%) who discontinued anakinra during the mean 3 years of follow-up, $28.1 \%$ discontinued due to complete remission (at mean follow-up at 4.7 years, 19 of 20 pts were still in remission, and 1 began anakinra again), $33.8 \%$ due to adverse events, $22.5 \%$ due to primary ineffectiveness, and $15.4 \%$ due to secondary ineffectiveness

Relative to BL, anakinra significantly $(p<0.0001)$ reduced mean Pouchot's scores ${ }^{\mathrm{b}}$, as well as specific AOSD symptoms (fever, rash, pneumonia, pericarditis, pleuritis, sore throat, lymphadenopathy, hepatomegaly, myalgia, arthritis and macrophage activation syndrome) and all laboratory manifestations (ESR and CRP, ferritin and liver enzyme levels) at 3, 6 and 12 months

Corticosteroid use decreased during anakinra treatment: the proportion of pts using corticosteroids at 1 year was significantly lower than that at BL (55.6 vs $97.8 \% ; p<0.001)$; the mean prednisolone-equivalent dosage at 3 months was significantly lower than that at BL (8.8 vs 77.6 $\mathrm{mg} /$ day; $p<0.0001$ ), with reductions to 5.2 and $3.4 \mathrm{mg} /$ day at 6 and 12 months, respectively

DMARD use also significantly decreased during anakinra treatment $(59.7 \%$ at 1 year vs $85.7 \%$ at $\mathrm{BL} ; p<0.001)$

$A O S D$ adult-onset Still's disease, $B L$ baseline, $C R P$ C-reactive protein, DMARD disease-modifying anti-rheumatic drug, ESR erythrocyte sedimentation rate, $p t$ patient, $R C T$ randomized, controlled trial, $S F-36$ Medical Outcomes Study Short-Form 36, $T D$ treatment difference

${ }^{a}$ Defined as being afebrile in the absence of NSAID treatment $24 \mathrm{~h}$ prior to measurement, with normal levels of CRP and ferritin, and normal swollen/tender joint counts

${ }^{\mathrm{b}}$ Considers 12 AOSD manifestations: fever (temperature $\geq 39{ }^{\circ} \mathrm{C}$ ), sore throat, myalgia, arthritis, pericarditis, hepatomegaly, evanescent rash, pleuritis, pneumonia, lymphadenopathy, hyperferritinaemia (ferritin $\geq 3000 \mathrm{ng} / \mathrm{mL}$ ), leucocytosis (white blood cell count $>15,000 / \mathrm{mm}^{3}$ )

The meta-analysis evaluated the efficacy of bDMARDs, in particular anakinra, in a total of 417 patients with AOSD in 19 studies (18 observational studies/case series and the anakinra open-label RCT [18]). Based on data in 271 anakinra recipients from 10 studies, complete remission was achieved by $72 \%$ (95\% CI 61-81; range 50-96\%) of patients, and clinical response (i.e. complete or partial remission) by $87 \%$ (95\% CI 79-93; range 50-97\%) of patients with AOSD [21]. The efficacy of IL-6 inhibitor tocilizumab (based on data from 73 patients in 6 studies) and TNF- $\alpha$ inhibitors (73 patients in 3 studies) [21] was also analysed. Overall, the efficacy of anakinra was broadly comparable to that of the IL- 6 inhibitor tocilizumab, and greater than that of TNF- $\alpha$ inhibitors [21]. However, these results were limited by the varying quality of the studies included in the meta-analysis, as well as the moderate-to-high between-study heterogeneity [21].

According to the literature review [22], IL-1 inhibitors (i.e. anakinra, rilonacept and canakinumab) had similar efficacy and provided better clinical outcomes than classical treatments (i.e. corticosteroids, NSAIDs and methotrexate), and also reduced the use of corticosteroids and DMARDs. 


\section{What is the efficacy of anakinra in systemic juvenile idiopathic arthritis?}

\section{Versus placebo in a double-blind trial and its open-label extension}

Treatment with subcutaneous anakinra was effective relative to placebo in patients with SJIA in a double-blind RCT (Table 3) [23]. The trial included 24 patients aged 2-20 years (mean 8.5 years) with SJIA of $>6$ months' duration, active systemic disease and significant overall disease activity at day 1. Current treatment with intravenous or intra-articular corticosteroids, immunosuppressive drugs and/or DMARDs was discontinued $\geq 1$ month before study onset; treatment with stable dosages of NSAIDs and oral corticosteroids (mean $0.59 \mathrm{mg} / \mathrm{kg}$ ) was allowed. Patients were randomized to receive either once-daily subcutaneous injections of anakinra $2 \mathrm{mg} / \mathrm{kg} /$ day (to a maximum of $100 \mathrm{mg} /$ day) or placebo for 1 month during the double-blind phase of the trial, followed by an open-label phase in which all patients received anakinra for up to 11 months [23].

Anakinra was significantly more effective than placebo with regard to the primary endpoint of the proportion of patients who had a modified American College of

Table 3 Efficacy of subcutaneous anakinra $2 \mathrm{mg} / \mathrm{kg} /$ day (maximum of $100 \mathrm{mg} /$ day) in treating patients with systemic juvenile idiopathic arthritis in a placebo-controlled trial, its open-label extension [23] and a cohort study [24]

Multicentre 1-month RCT in 24 pts aged 2-20 years with active SJIA of > 6 months' duration [23]

mACR-Pedi 30 response $^{\text {a }}$ (primary endpoint) achieved by significantly more anakinra than placebo recipients at 1 month $(67 \mathrm{vs} 8 \% ; p=0.003)$ Significantly ( $p \leq 0.04)$ more anakinra than placebo were ACR-Pedi-30 responders without fever (92 vs $50 \%$ ), ACR-Pedi-30 responders without fever and CRP $<15 \mathrm{mg} / \mathrm{L}$ ( 83 vs $25 \%$ ), mACR-Pedi ${ }^{\mathrm{a}} 50$ responders $\left(58\right.$ vs $0 \%$ ) and mACR-Pedi ${ }^{\mathrm{a}} 70$ responders $(42$ vs $0 \%)$

Significant TDs favouring anakinra over placebo were shown with regard to the mean decrease from BL in CRP and serum amyloid A levels, ESR, the number of joints with active disease and physician assessment of disease activity

No significant TDs were shown for mean changes from BL in the number of joints with limitations of passive motion, and scores for the Childhood Health Assessment Questionnaire, parent/pt global assessment and parent/pt assessment of pain

Open-label 11-month extension of RCT trial in 18 pts [23]

Of the 10 pts who switched from placebo to anakinra, 9 achieved mACR-Pedi 30 responses at 1 month

Of the 8 pts who responded to anakinra in the RCT and had their corticosteroid dosage reduced, 5 were still responders at month 2

In the $17 \mathrm{pts}$ in the trial at month 6 , the daily prednisolone-equivalent dosage was $0.18 \mathrm{mg} / \mathrm{kg}$ (range $0-0.58$ )

In the $6 \mathrm{mACR}$-Pedi-30 responders at month 6 , the mean prednisolone-equivalent daily dosage was lower than at BL $(0.3 \mathrm{vs} 0.51 \mathrm{mg} / \mathrm{kg})$; all of these pts had an ACR-Pedi-30, -50 or -70 response to anakinra at month 1

At 11 months, 16 pts remained in the trial, including 7 responders ( 6 had discontinued corticosteroid treatment and 5 had inactive SJIA)

First-line DMARD treatment in 20 pts with new-onset SIJA in a single-centre cohort study [24]

Normal body temperatures were achieved by $90 \%$ of pts within 3 days; $80 \%$ of pts achieved normal ESR values and CRP and ferritin levels within 1 month, with normalization being maintained for up to 3 years

aACR-Pedi- 90 responses $^{\text {b }}$ were achieved by $80 \%$ of pts by 1 month, and by $85 \%$ at 1 year (all of whom also met the criteria for clinically inactive SJIA ${ }^{\mathrm{c}}$ )

Of the 13 pts who achieved clinically inactive SJIA ${ }^{c}$ with anakinra monotherapy, 11 were able to taper and discontinue anakinra within year 1 of treatment

The 7 pts with an incomplete response to anakinra monotherapy during year 1 required the addition of a corticosteroid and/or a DMARD or a switch to another DMARD to achieve a response

If a relapse occurred during tapering of anakinra, treatment could often be restarted, then successfully tapered and stopped

At 2 year follow-up, aACR-Pedi- $90^{\mathrm{b}}$ responses were sustained by 12 of 14 pts $(86 \%)$; all of these pts met the criteria for clinical remission ${ }^{\mathrm{d}}$, regardless of current use $(n=4)$ or non-use of medication $(n=8)$

At 3 year follow-up, aACR-Pedi- $90^{\mathrm{b}}$ responses were sustained by 10 of 11 pts (91\%); all of these pts met the criteria for clinical remission ${ }^{\mathrm{d}}$, regardless of current use $(n=2)$ or non-use of medication $(n=8)$

$a A C R$-Pedi adapted ACR-Pedi, ACR-Pedi American College of Rheumatology Pediatric, $B L$ baseline, $C R P$ C-reactive protein, $D M A R D$ diseasemodifying anti-rheumatic drug, ESR erythrocyte sedimentation rate, mACR-Pedi modified ACR-Pedi, $p t$ patient, $R C T$ randomized, controlled trial, SIJA systemic juvenile idiopathic arthritis, $T D$ treatment difference

${ }^{a}$ mACR-Pedi $x$ response: defined as an ACR-Pedi $x$ response, an absence of SIJA-related fever (no body temperature $\geq 38^{\circ} \mathrm{C}$ over the past 8 days) and either a 50\% decrease from baseline or normalized values for both CRP and ESR

baACR-Pedi $x$ : defined as absence of fever and $\geq x$ improvement from BL in $\geq 3$ of the 6 core ACR-Pedi variables, with $\leq 1$ of the remaining variables worsening by $>30 \%$

${ }^{c}$ Defined as no active arthritis, no systemic features, no uveitis, a normal ESR ( $\left.\leq 20 \mathrm{~mm} / \mathrm{h}\right)$, and a physician global assessment indicating no disease activity (score of $\leq 10 \mathrm{~mm}$ on a 100 -mm visual analogue scale)

${ }^{\mathrm{d}}$ Defined as inactive disease for $\geq 6$ months during ongoing use of medication or $\geq 12$ months without the use of medication 
Rheumatology Pediatric (mACR-Pedi) 30 response to treatment at 1 month (Table 3) [23]. Significant treatment differences favouring anakinra over placebo at 1 month were also shown for most other response-based outcomes, including the proportion of ACR-Pedi 30 responders without fever, or without fever and CRP $<15 \mathrm{mg} / \mathrm{L}$, as well as the proportion of mACR-Pedi 50 and 70 responders (Table 3) [23]. No patients in either group achieved a mACR-Pedi-100 response [23]. Mean changes from baseline in some, but not all, SIJArelated outcomes and scores were also significantly greater with anakinra than with placebo at 1 month (Table 3) [23].

In the open-label phase, almost all patients who switched from placebo to anakinra achieved a mACR-Pedi 30 response after 1 month of anakinra (Table 3) [23]. Dosages of oral corticosteroids decreased or remained stable in the open-label extension, with many anakinra responders being able to discontinue corticosteroid treatment (Table 3) [23].

\section{As first-line DMARD treatment}

First-line treatment with anakinra $2 \mathrm{mg} / \mathrm{kg} /$ day (maximum of $100 \mathrm{mg} /$ day) effectively treated SJIA in the vast majority of patients in a prospective single-centre cohort study (Table 3) [24]. The study included 20 patients (mean age 7.9 years; range 1.1-15.3) with new-onset SJIA who had failed to respond to an NSAID (indomethacin), but who had not yet been treated with other DMARDs (including bDMARDs) or systemic corticosteroids.

Anakinra treatment was very effective as first-line DMARD treatment in this patient cohort. Normalization of body temperatures was shown in $90 \%$ of patients within 3 days, normalization of ESR, CRP and ferritin levels, and achievement of adjusted ACR-Pedi (aACR-Pedi) 90 responses in $80 \%$ of patients within 1 month and $85 \%$ at 1 year (Table 3) [24]. In this study, it was also possible to: taper and discontinue anakinra in patients who responded to treatment; restart, then taper and discontinue anakinra if a relapse in symptoms occurred after discontinuation; add a corticosteroid and/or a DMARD, or switch to another DMARD with an adequate response to anakinra monotherapy (Table 3) [24].

Sustained remission rates were high at 2 and 3 years follow-up (Table 3). All of the patients with aACR-Pedi 90 responses met the criteria for clinical remission, regardless of whether they were currently receiving or not receiving anakinra (Table 3) [24].

First-line treatment with anakinra also provided rapid and sustained complete or partial responses for most, but not all, patients with SJIA according to a retrospective study in 46 patients [25]. Some patients required the addition of corticosteroids or DMARDs to achieve a response, and anakinra could generally be discontinued in responders and restarted when a relapse occurred [25].

\section{Retrospective observational studies}

According to a 2010 systematic review of case series in a total of 140 patients with SJIA and poor expected outcomes, anakinra resolved systemic and laboratory manifestations of SJIA in almost all $(\approx 95 \%)$ patients, generally within 1 week [26]. Anakinra was used with a variety of concomitant agents, including corticosteroids, NSAIDs and DMARDs, with anakinra allowing corticosteroid treatment to be reduced or discontinued. Anakinra resolved systemic symptoms better than articular symptoms, and was more effective in patients with fewer affected joints and shorter disease duration. Treatment with anakinra was discontinued after response in some patients, and due to primary or secondary inefficacy or tolerability issues in other patients [26].

Similar findings are described in a retrospective study in 25 patients with SJIA who received anakinra for $\geq 6$ months [27]. Treatment with anakinra was associated with inactive disease in 14 patients $(56 \%)$, with these patients having a rapid response (median time to inactive disease of 2.1 months), which was sustained at last follow-up (median follow-up duration 2.8 years). Anakinra was discontinued in 9 of the 14 patients, and was continued as monotherapy in the remaining 5 patients [27]. Treatment with anakinra was corticosteroid-sparing. In patients receiving corticosteroids at baseline, corticosteroid therapy was discontinued in all 6 of the responders, and 4 of the 8 non-responders. The remaining 4 non-responders received a mean prednisoneequivalent dosage of $0.04 \mathrm{mg} / \mathrm{kg} / \mathrm{day}$ [27].

\section{Relative to other biological DMARDs}

Anakinra and tocilizumab provided similar clinical responses at 1 year in 76 patients with SJIA in a UK registry study [28]. At 1 year in the 22 anakinra and 54 tocilizumab recipients, an ACR-Pedi-90 response was achieved by 31 and $46 \%$ of patients, respectively, minimal disease activity in 49 and 52\%, and clinically inactive disease in 25 and $45 \%$, with no significant between-treatment differences [28].

In a registry study in 245 patients with SJIA in Germany, treatment with an IL-1 inhibitor [anakinra $(n=38)$ or canakinumab $(n=22)$ ] or tocilizumab $(n=71)$ was more effective than treatment with etanercept $(n=143)$ [29]. At 6 months, significantly more IL-1 inhibitor $(p<0.001)$ and tocilizumab $(p \leq 0.05)$ recipients than etanercept recipients achieved a Juvenile Disease Activity Score 10 (JADAS10) score of $\leq 1$ (i.e. remission) [ 52 and 36.4 vs $20 \%$ ], or a JADAS 10 score of $\leq 3.8$ (i.e. minimal disease activity) [ 68 and 61 vs $33 \%$ ]. At 2 years, a JADAS 10 score of $\leq 1$ was achieved by 52,37 
and $20 \%$ of IL-1 inhibitor, tocilizumab and etanercept recipients, respectively, and a JADAS 10 score of $\leq 3.8$ by 68,61 and $35 \%$, and ACR inactive disease by 56,33 and $24 \%$. Starting treatment within 1 year of diagnosis instead of starting it later significantly improved the likelihood of JADAS remission with IL-1 inhibitors [odds ratio (OR) 6.6; $p=0.002$ ] and tocilizumab (OR 3.9; $p=0.012$ ). There were no significant differences in outcomes between IL-1 inhibitors and tocilizumab [29].

\section{Network meta-analyses}

Network meta-analyses based on indirect evidence found no differences between the efficacy of anakinra and other bDMARDs in the treatment of SJIA [30, 31]. Based on data from 1 RCT each for anakinra, canakinumab and tocilizumab versus placebo ( $n=24,84$ and 112, respectively), the likelihood of achieving a mACR-Pedi 30 response with anakinra did not differ significantly from that with canakinumab or tocilizumab [30].

The second network meta-analysis of bDMARDs in SJIA included the same 3 RCTs for anakinra, canakinumab and tocilizumab, plus 2 placebo-controlled trials of rilonacept in a total of 95 patients [31]. In pair-wise comparisons, the efficacy of anakinra with regard to achieving a mACR-Pedi 30 response did not differ to a significantly greater extent than that of any of the comparator bDMARDs (i.e. canakinumab, tocilizumab and rilonacept); however, the efficacy of canakinumab and tocilizumab was significantly greater than that of rilonacept [31].

These analyses are limited by the paucity of clinical data, short study duration, high between-RCT heterogeneity and varying study quality [30,31], as well as the use of some data derived from abstract reports [30].

\section{What is the safety profile of anakinra in Still's disease?}

Due to the small number of patients in the key clinical trials of anakinra in Still's disease, as well as the retrospective design of almost all observational studies, it is difficult to determine the overall safety of anakinra specifically in this patient population. According to the EU summary of product characteristics (SPC) [15], the safety profile of anakinra in patients with AOSD and SJIA is consistent with its safety profile in patients with RA, with the exception of risk of MAS [15]. Patients with Still's disease may spontaneously develop MAS, including during treatment with anakinra; importantly, no causal relationship between MAS and treatment with anakinra has been established [15].

\section{General tolerability}

According to the EU SPC [15]; the following adverse events (AEs) are associated with anakinra treatment (regardless of indication):

- Very common AEs (i.e. occur in $\geq 10 \%$ of recipients) Headache, injection-site reactions (ISRs) and increased blood cholesterol levels.

- Common AEs (i.e. occur in $\geq 1$ to $<10 \%$ of recipients) Serious infections, neutropenia and thrombocytopenia

- Uncommon AEs (i.e. occur in $\geq 0.1$ to $<1 \%$ of recipients) Allergic reactions (e.g. anaphylactic reactions, angioedema, urticaria and pruritus), increased hepatic enzyme levels and rash.

No serious treatment-emergent AEs (TEAEs), with the exception of 1 disease flare, were reported in the RCT and its extension in patients with AOSD [18], or in the cohort study in patients with SJIA [24]. In the 1-month RCT in 24 patients with SJIA, 14 TEAEs were reported with anakinra and 13 with placebo [23]. In the 11-month extension, 89 TEAEs were reported in the 22 anakinra recipients, with serious TEAEs in 6 patients (4 cases of infection and 1 of vertebral collapse were successfully resolved and the patients continued anakinra treatment; the remaining patient was diagnosed with Crohn's disease and discontinued anakinra) [23].

Data comparing the safety of anakinra relative to other bDMARDs are limited. In the UK registry study of bDMARDs in patients with SJIA, discontinuation of treatment was significantly $(p=0.002)$ more common with anakinra than with tocilizumab, with $41 \%$ of anakinra and $11 \%$ of tocilizumab recipients discontinuing treatment for any reason by 1 year [28]. Of the 9 patients who discontinued anakinra, 1 stopped because of remission, 4 due to inefficacy and 4 due to TEAEs ( 1 with stomach cramps and diarrhoea, 1 with ISR, and 2 with difficulties with daily injection). Of the 6 patients who discontinued tocilizumab, 2 stopped due to inefficacy, 3 due to TEAEs ( 1 each with rash, neutropenia and active MAS), and 1 due to unknown reasons [28]. In the German registry study, the risk of serious TEAEs was significantly higher with IL-1 inhibitors than with etanercept (risk ratio 2.9; $p<0.01$ ) [29].

\section{Injection-site reactions}

ISRs (including erythema, ecchymosis, inflammation and pain) are the most common and consistently reported treatment-related AEs (TRAEs) [15]. For example, in patients with RA, $71 \%$ of recipients of anakinra $100 \mathrm{mg} /$ day experienced ISRs compared with $28 \%$ of placebo recipients; almost all (95\%) of ISRs were mild to moderate in severity. 
ISRs generally appear within 2 weeks of therapy, disappear within 4-6 weeks, and are uncommon after 1 month of therapy if not previously experienced. Discomfort and ISRs can be alleviated by taking precautions before and after administration of the injection (Table 4) [15].

ISRs were also the most common TRAEs in the key clinical trials of anakinra in Still's disease [18, 23, 24]. In patients with AOSD, ISRs were reported during the initial 24-week RCT in 8 of 12 anakinra recipients (7 with grade 1 and 1 with grade 2 ISRs) and in 4 additional patients during the 28-week extension; none of these patients discontinued treatment because of an ISR [18].

In the 1-month RCT in 24 patients with SJIA, the most common TRAEs with both anakinra and placebo were pain at injection ( 8 with anakinra and 6 with placebo) and post-injection erythema ( 3 and 1 , respectively) [23]. In the 11-month open-label extension in 22 patients, there were 15 reports of pain at injection and 6 reports of post-injection erythema [23]. Local skin reactions were reported in 13 of 20 patients in the cohort study [24]. According to data from the EU SPC, 1 of the 15 patients with SJIA followed for up to 1.5 years discontinued anakinra due to ISRs [15].

\section{Other adverse events of special interest}

Serious infections may occur in patients receiving bDMARDs [32], and appropriate precautions (Table 4) should be followed [15]. In a US database study, the risk of infection was higher in 247 patients with juvenile idiopathic arthritis (most of whom had SJIA) receiving anakinra than in 160 patients with SJIA receiving methotrexate (adjusted hazard ratio 3.5 ; 95\% CI 1.8-6.8); however, treatment differences in disease phenotype may have confounded the results [32]. The only serious infection in the 15 SJIA patients followed for up to 1.5 years was 1 report of serious hepatitis in connection with a cytomegalovirus infection [15].

Other precautions should also be followed with regard to the risk of potential hepatic events, allergic reactions and neutropenia (Table 4) [15]. The use of anakinra is not recommended in patients with a pre-existing malignancy, as anakinra has immunosuppressive properties and data are lacking in this patient population (Table 4) [15]. Although neutralizing anti-anakinra antibodies have been reported during anakinra treatment; they are typically transient and are not associated with AEs or reductions in efficacy [15].

Table 4 Special warnings and precautions related to the use of subcutaneous anakinra in the treatment of Still's disease in the EU [15]

What precautions should be taken to alleviate injection-site discomfort and reactions?

Before the injection Inject at alternating injection sites

Cool the injection site with cold packs; allow the pre-filled syringe to reach room temperature or hold it in one's hand for a few minutes

After the injection Cool the injection site with cold packs and apply topical corticosteroids/antihistamines

What precautions should be taken with regard to infections?

Active infection at initiation

Do not start treatment with anakinra until infection is resolved

Serious infection develops

Monitor carefully if anakinra is continued (data regarding discontinuing/continuing anakinra during serious infections in pts with Still's disease are limited)

Tuberculosis

Screen pts for latent tuberculosis before initiating anakinra, taking medical guidelines into account

Reactivation of hepatitis B Screen for viral hepatitis before initiating anakinra, taking medical guidelines into account

What precautions should be taken with regard to the risk of hepatic events?

Monitor liver function Consider routine monitoring of hepatic enzymes during the first month of anakinra treatment (increases in hepatic enzymes and acute hepatitis have reported in pts with Still's disease, predominantly during the first month of treatment)

Monitoring is especially important in pts with pre-dosing factors for hepatic events or who develop liver dysfunction symptoms

What precautions should be taken with regard to the risk of neutropenia?

Monitor ANCs Assess prior to starting treatment, monthly during the first 6 months of treatment, then quarterly

Do not initiate anakinra in pts with neutropenia

Pts with neutropenia $\quad \mathrm{ANC}<1.5 \times 10^{9} / \mathrm{L}$ prior to starting treatment: do not initiate anakinra

ANC $<1.5 \times 10^{9} / \mathrm{L}$ during treatment: monitor ANCs closely and discontinue anakinra

What other special warnings/precautions pertain to the use of anakinra?

Allergic and hypersensitivity reactions

Immunosuppression
Pts with hypersensitivity to anakinra, any excipient in the injection solution or Escherichia coli-derived proteins prior to starting treatment: use of anakinra is contraindicated

Pts with severe allergic reactions during treatment: discontinue anakinra

Pts with pre-existing malignancy: use of anakinra is not recommended (lack of data)

$A N C$ absolute neutrophil count, pts patients 


\section{What is the current clinical position of anakinra in Still's disease?}

The extended EU approval of anakinra to include the treatment of Still's disease (AOSD and SJIA) provides an important option in the treatment of this rare and disabling inflammatory disorder. Anakinra is a rational treatment for Still's disease, including AOSD and SJIA, as it:

- Targets IL-1 induced inflammation As anakinra is an IL-1 inhibitor, it is active against the high levels of proinflammatory cytokine IL-1 associated with the systemic manifestations of these disorders. Responses to anakinra seem to be better in patients with predominantly systemic manifestations (which are associated with high levels of IL-1) rather than chronic articular manifestations (which are associated with high levels of TNF- $\alpha$ ).

- Improves the clinical and laboratory manifestations of these disorders Many patients achieve clinical remission and response, with rapid and sustained improvements in new and refractory AOSD- and SJIA-induced symptoms and normalization of laboratory values.

- Is corticosteroid-sparing Improvements in the signs and symptoms of new-onset or refractory AOSD and SJIA allows concomitant exposure to corticosteroids to be avoided, reduced or discontinued.

- Has an acceptable safety profile The safety profile of anakinra in patients with AOSD and SJIA is consistent with its safety profile in patients with RA and CAPS, with ISRs being the most common TRAE. Appropriate precautions should be taken to reduce the likelihood of ISRs, serious infections, neutropenia and other adverse drug reactions.

MAS is a difficult-to-treat potential complication of Still's disease. However, limited evidence suggests that anakinrabased treatment may provide rapid clinical improvements in MAS in patients with AOSD [20,33-35] or SJIA [33, 36-38]. MAS should be diagnosed and treated as soon as possible, with attention being paid to the presence of symptoms of infection or worsening Still's disease, both of which are known to trigger MAS [15]. Of note, MAS may occur during anakinra treatment in patients with AOSD or SJIA, but a relationship between the use of anakinra and the development of MAS has not been established [15].

Further information on the efficacy, safety, pharmacokinetic, and immunogenicity profiles of anakinra 2 and $4 \mathrm{mg} /$ $\mathrm{kg} /$ day (respective maximums of 100 and $200 \mathrm{mg} /$ day) versus placebo will be provided by an ongoing 12 -week phase $3 \mathrm{RCT}$ in $\approx 81$ adult/paediatric patients with AOSD or SJIA [39].
Head-to-head RCTs comparing the efficacy of anakinra with that of other DMARDs, particularly other bDMARDs, would help clarify the relative position of such agents in treatment of these conditions. However, such RCTs are unlikely to be conducted due to the rarity of AOSD and SJIA. Indirect comparisons, therefore, have been used to evaluate the efficacy of anakinra relative to other agents in the treatment of AOSD [21, 22] and SJIA [30, 31]. Although limited by between-study differences in patient populations (partially due to differing inclusion/exclusion criteria), the following overall results have been reported in a literature review [22], a meta-analysis [21] and network meta-analyses [30, 31]:

- The efficacy of anakinra in treating AOSD is similar to that of other IL-1 inhibitors (canakinumab and rilonacept) [22], broadly comparable to that with the IL-6 inhibitor tocilizumab [21], and greater than that of TNF- $\alpha$ inhibitors [21] and classic DMARDs (e.g. corticosteroids, NSAIDs and methotrexate) [22].

- The efficacy of anakinra in treating SJIAs is similar to that of canakinumab [30,31], rilonacept [31] and tocilizumab [30, 31].

Further network meta-analyses of the use of various bDMARDs in the treatment of AOSD and SJIA, as well as pharmacoeconomic analyses of their relative cost-utility, would be of interest.

Acknowledgements The manuscript was reviewed by: S. Castañeda, Department of Rheumatology, Hospital Universitario La Princesa, Madrid, Spain; T. Pettersson, Department of Medicine, Helsinki University Central Hospital and Helsinki University, Helsinki Finland. During the peer review process, Swedish Orphan Biovitrum AB, the marketing-authorization holder of anakinra, was also offered an opportunity to provide a scientific accuracy review of their data. Changes resulting from comments received were made on the basis of scientific and editorial merit.

\section{Compliance with ethical standards}

Funding The preparation of this review was not supported by any external funding.

Conflicts of interest K.A. Lyseng-Williamson is an employee of Adis/ Springer, is responsible for the article content and declares no conflicts of interest.

Open Access This article is distributed under the terms of the Creative Commons Attribution-NonCommercial 4.0 International License (http://creativecommons.org/licenses/by-nc/4.0/), which permits any noncommercial use, distribution, and reproduction in any medium, provided you give appropriate credit to the original author(s) and the source, provide a link to the Creative Commons license, and indicate if changes were made. 


\section{References}

1. Sfriso P, Bindoli S, Galozzi P. Adult-onset Still's disease: molecular pathophysiology and therapeutic advances. Drugs. 2018;78(12):1187-95.

2. Maria AT, Le Quellec A, Jorgensen C, et al. Adult onset Still's disease (AOSD) in the era of biologic therapies: dichotomous view for cytokine and clinical expressions. Autoimmun Rev. 2014;13(11):1149-59.

3. Zhou S, Qiao J, Bai J, et al. Biological therapy of traditional therapy-resistant adult-onset Still's disease: an evidence-based review. Ther Clin Risk Manag. 2018;14:167-71.

4. Sfriso P, Priori R, Valesini G, et al. Adult onset Still's disease: an Italian multicenter retrospective observational study of manifestations and treatments in 245 patients. Clin Rheumatol. 2016;35(7):1683-9.

5. Castañeda S, Blanco R, González-Gay MA. Adult-onset Still's disease: advances in the treatment. Best Pract Res Clin Rheumatol. 2016;30(2):222-38.

6. Pascual V, Allantaz F, Arce E, et al. Role of interleukin-1 (IL1) in the pathogenesis of systemic onset juvenile idiopathic arthritis and clinical response to IL-1 blockade. J Exp Med. 2005;201(9):1479-86.

7. Arthur VL, Shuldiner E, Remmers EF, et al. $I L 1 R N$ variation influences both disease susceptibility and response to recombinant human interleukin-1 receptor antagonist therapy in systemic juvenile idiopathic arthritis. Arthritis Rheumatol. 2018;70(8):1319-30.

8. Kimura Y, Weiss JE, Haroldson KL, et al. Pulmonary hypertension and other potentially fatal pulmonary complications in systemic juvenile idiopathic arthritis. Arthritis Care Res (Hoboken). 2013;65(5):745-52.

9. Nirmala N, Brachat A, Feist E, et al. Gene-expression analysis of adult-onset Still's disease and systemic juvenile idiopathic arthritis is consistent with a continuum of a single disease entity. Pediatr Rheumatol Online J. 2015;13:50.

10. Néel A, Wahbi A, Tessoulin B, et al. Diagnostic and management of life-threatening adult-onset Still disease: a French nationwide multicenter study and systematic literature review. Crit Care. 2018;22(1):88.

11. Ruscitti P, Cipriani P, Masedu F, et al. Adult-onset Still's disease: evaluation of prognostic tools and validation of the systemic score by analysis of 100 cases from three centers. BMC Med. 2016;14(1):194

12. Schulert GS, Grom AA. Pathogenesis of macrophage activation syndrome and potential for cytokine-directed therapies. Annu Rev Med. 2015;66:145-59.

13. Bracaglia C, Prencipe G, De Benedetti F. Macrophage activation syndrome: different mechanisms leading to a one clinical syndrome. Pediatr Rheumatol. 2017;15(1):5.

14. Ringold S, Weiss PF, Beukelman T, et al. 2013 update of the 2011 American College of Rheumatology recommendations for the treatment of juvenile idiopathic arthritis: recommendations for the medical therapy of children with systemic juvenile idiopathic arthritis and tuberculosis screening among children receiving biologic medications. Arthritis Rheum. 2013;65(10):2499-512.

15. Kineret (anakinra) $100 \mathrm{mg} / 0.67 \mathrm{ml}$ solution for injection in prefilled syringe. London: European Medicines Agency; 2018.

16. ter Haar NM, Tak T, Mokry M, et al. Reversal of sepsis-like features of neutrophils by interleukin-1 blockade in patients with systemic-onset juvenile idiopathic arthritis. Arthritis Rheumatol. 2018;70(6):943-56.

17. Jeyaratnam J, ter Haar NM, Lachmann HJ, et al. The safety of liveattenuated vaccines in patients using IL-1 or IL-6 blockade: an international survey. Pediatric Rheumatology. 2018;16(19):1-6.
18. Nordström D, Knight A, Luukkainen R, et al. Beneficial effect of interleukin 1 inhibition with anakinra in adult-onset Still's disease: an open, randomized, multicenter study. J Rheumatol. 2012;39(10):2008-11.

19. Ortiz-Sanjuán F, Blanco R, Riancho-Zarrabeitia L, et al. Efficacy of anakinra in refractory adult-onset Still's disease: multicenter study of 41 patients and literature review. Medicine (Baltimore). 2015;94(39):e1554.

20. Colafrancesco S, Priori R, Valesini G, et al. Response to interleukin-1 inhibitors in 140 Italian patients with adult-onset Still's disease: a multicentre retrospective observational study. Front Pharmacol. 2017;8:369.

21. Ruscitti P, Ursini F, Cipriani P, et al. Biologic drugs in adult onset Still's disease: a systematic review and meta-analysis of observational studies. Expert Rev Clin Immunol. 2017;13(11):1089-97.

22. Junge G, Mason J, Feist E. Adult onset Still's disease: the evidence that anti-interleukin-1 treatment is effective and well-tolerated (a comprehensive literature review). Semin Arthritis Rheum. 2017;47(2):295-302.

23. Quartier P, Allantaz F, Cimaz R, et al. A multicentre, randomised, double-blind, placebo-controlled trial with the interleukin-1 receptor antagonist anakinra in patients with systemic-onset juvenile idiopathic arthritis (ANAJIS trial). Ann Rheum Dis. 2011;70(5):747-54.

24. Vastert SJ, de Jager W, Noordman BJ, et al. Effectiveness of firstline treatment with recombinant interleukin-1 receptor antagonist in steroid-naive patients with new-onset systemic juvenile idiopathic arthritis: results of a prospective cohort study. Arthritis Rheumatol. 2014;66(4):1034-43.

25. Nigrovic PA, Mannion M, Prince FH, et al. Anakinra as first-line disease-modifying therapy in systemic juvenile idiopathic arthritis: report of forty-six patients from an international multicenter series. Arthritis Rheum. 2011;63(2):545-55.

26. Swart JF, Barug D, Möhlmann M, et al. The efficacy and safety of interleukin-1-receptor antagonist anakinra in the treatment of systemic juvenile idiopathic arthritis. Expert Opin Biol Ther. 2010;10(12):1743-52.

27. Pardeo M, Pires Marafon D, Insalaco A, et al. Anakinra in systemic juvenile idiopathic arthritis: a single-center experience. $\mathrm{J}$ Rheumatol. 2015;42(8):1523-7.

28. Kearsley-Fleet L, Beresford MW, Davies R. Short-term outcomes in patients with systemic juvenile idiopathic arthritis treated with either tocilizumab or anakinra. Rheumatology (Oxford). Epub 2018. https://doi.org/10.1093/rheumatology/key262. 2017

29. Horneff G, Schulz AC, Klotsche J, et al. Experience with etanercept, tocilizumab and interleukin-1 inhibitors in systemic onset juvenile idiopathic arthritis patients from the BIKER registry. Arthritis Res Ther. 2017;19(1):256.

30. Otten MH, Anink J, Spronk S, et al. Efficacy of biological agents in juvenile idiopathic arthritis: a systematic review using indirect comparisons. Ann Rheum Dis. 2013;72(11):1806-12.

31. Tarp S, Amarilyo G, Foeldvari I, et al. Efficacy and safety of biological agents for systemic juvenile idiopathic arthritis: a systematic review and meta-analysis of randomized trials. Rheumatology (Oxford). 2016;55(4):669-79.

32. Beukelman T, Xie F, Baddley JW, et al. The risk of hospitalized infection following initiation of biologic agents versus methotrexate in the treatment of juvenile idiopathic arthritis. Arthritis Res Ther. 2016;18(1):210.

33. Sönmez HE, Demir S, Bilginer Y, et al. Anakinra treatment in macrophage activation syndrome: a single center experience and systemic review of literature. Clin Rheumatol. Epub 2018. https ://doi.org/10.1007/s10067-018-4095-1.

34. Mehta MV, Manson DK, Horn EM, et al. An atypical presentation of adult-onset Still's disease complicated by pulmonary hypertension and macrophage activation syndrome treated with 
immunosuppression: a case-based review of the literature. Pulm Circ. 2016;6(1):136-42.

35. Lenert A, Yao Q. Macrophage activation syndrome complicating adult onset Still's disease: a single center case series and comparison with literature. Semin Arthritis Rheum. 2016;45(6):711-6.

36. Kelly A, Ramanan AV. A case of macrophage activation syndrome successfully treated with anakinra. Nat Clin Pract Rheumatol. 2008;4(11):615-20.

37. Rajasekaran S, Kruse K, Kovey K, et al. Therapeutic role of anakinra, an interleukin-1 receptor antagonist, in the management of secondary hemophagocytic lymphohistiocytosis/sepsis/multiple organ dysfunction/macrophage activating syndrome in critically ill children. Pediatr Crit Care Med. 2014;15(5):401-8.

38. Miettunen PM, Narendran A, Jayanthan A, et al. Successful treatment of severe paediatric rheumatic disease-associated macrophage activation syndrome with interleukin- 1 inhibition following conventional immunosuppressive therapy: case series with 12 patients. Rheumatology (Oxford). 2011;50(2):417-9.

39. Swedish Orphan Biovitrum. A study to evaluate efficacy and safety of anakinra in the treatment of Still's disease (SJIA and AOSD) (anaSTILLs). [ClinicalTrials.gov identifier NCT03265132].https:// clinicaltrials.gov/ct2/show/NCT03265132. Accessed 12 Sep 2018 